\title{
Spectroscopy of New Faint Planetary Nebulae
}

\author{
F. Kerber ${ }^{1}$, H. Gröbner ${ }^{1}$, R. Weinberger ${ }^{1}$ and M. Roth ${ }^{2}$ \\ ${ }^{1}$ Institut für Astronomie, Universität Innsbruck; \\ ${ }^{2}$ Las Campanas Observatory, Carnegie Institution of Washington, Chile
}

Our department has a long-standing tradition of discovery of planetary nebulae (PN), see e.g. Tamura \& Weinberger (A\&A 298, 204 (1995)) and Kerber et al. (A\&AS in press (1996)) for the latest contributions.

Here we present our most recent additions: The following nine newly detected PNe have been observed in April 1996 with the 2.5-m duPont Telescope at Las Campanas Observatory, Chile. Operating the modular spectrograph we covered the spectral range from $4800 \AA$ to $6800 \AA$. The spectral resolution was $2 \AA /$ pixel.

All objects have been discovered on ESO/SERC films by visual inspection. Some have been found during our ongoing dedicated search for PNe, others have been discovered as a byproduct during a search for galaxies near the galactic plane, the so-called Zone of Avoidance (ZoA), see e.g. Lercher et al. (A\&AS 117, 369 (1996)). All objects are faint evolved PNe of low surface brightness, some of them are decidedly asymmetric and do not look like a typical PN. In about one third of the sample indications for interaction with the interstellar medium (ISM) can be discerned. Such indications are asymmetric brightness distribution, particularily pronounced in the low ionsation stages like [N II], and a decentrally located central star. Most of the examples for interaction with the ISM are very large nebulae, see Kwitter \& Tweedy (AJ 108, 188 (1994)) and Xilouris et al. (A\&A 310, 603 (1996)). Most of our examples are much smaller and therefore suited for further study with fields of view provided by normal CCDs. For details of the theoretical aspects of the interaction process see Borkowski et al. (ApJ 360, 173 (1990)) and Soker at al. (AJ 102, 138 (1991)). This process seems to be much more common than once thought, since more and more examples are discovered. Apparently interaction with the ISM is an important aspect of late stages of PN evolution that certainly deserves more study.

\begin{tabular}{clllcc}
\hline Name & \multicolumn{1}{c}{ Designation } & RA (2000.0) & DEC (2000.0) & $\oslash\left[^{\prime \prime}\right]$ & ESO-R \\
\hline KeWe 2 & PN G228.5-11.4 & $06^{\mathrm{h}} 37^{\mathrm{m}} 39.3^{\mathrm{s}}$ & $-18^{\circ} 57^{\prime} 30^{\prime \prime}$ & 30 & 557 \\
KeWe 3 & PN G238.4-01.8 & $07^{\mathrm{h}} 33^{\mathrm{m}} 25.9^{\mathrm{s}}$ & $-23^{\circ} 26^{\prime} 08^{\prime \prime}$ & 120 & 492 \\
KeWe 4 & PN G257.8-05.4 & $08^{\mathrm{h}} 05^{\mathrm{m}} 33.7^{\mathrm{s}}$ & $-41^{\circ} 56^{\prime} 31^{\prime \prime}$ & 45 & 312 \\
NeVe 3-1 & PN G275.9-01.0 & $09^{\mathrm{h}} 34^{\mathrm{m}} 04^{\mathrm{s}}$ & $-53^{\circ} 12^{\prime} 00^{\prime \prime}$ & 40 & 166 \\
WeKG 1 & PN G307.5-03.6 & $13^{\mathrm{h}} 37^{\mathrm{m}} 32 \mathrm{~s} .7$ & $-66^{\circ} 08^{\prime} 28^{\prime \prime}$ & 15 & 096 \\
WeKG 2 & PN G308.4+00.4 & $13^{\mathrm{h}} 38^{\mathrm{m}} 41 \mathrm{~s} .7$ & $-61^{\circ} 55^{\prime} 51^{\prime \prime}$ & 35 & 133 \\
WeKG 3 & PN G310.6+01.4 & $13^{\mathrm{h}} 54^{\mathrm{m}} 25 \mathrm{~s} .6$ & $-60^{\circ} 27^{\prime} 20^{\prime \prime}$ & $13 \times 20$ & 132 \\
NeVe 3-2 & PN G326.4+07.0 & $15^{\mathrm{h}} 19^{\mathrm{m}} 43^{\mathrm{s}}$ & $-49^{\circ} 00^{\prime} 00^{\prime \prime}$ & 30 & 224 \\
KeWe 5 & PN G348.9+04.6 & $16^{\mathrm{h}} 57^{\mathrm{m}} 56.5^{\mathrm{s}}$ & $-35^{\circ} 24^{\prime} 41^{\prime \prime}$ & 15 & 391 \\
\hline
\end{tabular}

This research was supported by the "Fonds zur Förderung der wissenschaftlichen Forschung", project no. P10279-AST and by a travel grant from the Austrian "Bundesministerium für Wissenschaft, Forschung und Verkehr". This research has made use of the SIMBAD database, operated at CDS, Strasbourg, France. 